\title{
Error correction of a phase-only computer-generated hologram for an aspheric surface
}

H. Zhang

Y. Yan

H. Zhou

Y. J. Qiao

J. Si

D. Wang
School of Mechanical \& Power Engineering, Harbin University of Science and Technology, Harbin, China

School of Mechanical \& Power Engineering, Harbin University of Science and Technology, Harbin, China

School of Mechanical \& Power Engineering, Harbin University of Science and Technology, Harbin, China

School of Mechanical \& Power Engineering, Harbin University of Science and Technology, Harbin, China

School of Mechanical \& Power Engineering, Harbin University of Science and Technology, Harbin, China

Department of Physics and Optoelectronics, Taiyuan University of Technology, Taiyuan, China

When applying phase-only computer-generated hologram (CGH) as a standard model of optical measurement in computer-generated holography for aspheric surface testing, it has the advantage of simplifying optical path configuration and improving the diffraction efficiency of the incoming light. However, errors always exists during the encoding process of fabricating multiphase level CGHs and this kind of errors will be amplified level by level in the measurement. According to the analysis of the encoding error, the error of CGH increases linearly when its quantified period increases. For example, if the quantified period is 32, the maximum of encoding error is 16.46 which can lead wave-front aberration $0.085 \lambda$ of a secondary parabolic surface with $512 \times 512$ sampling pixels. In this article, an optimization method based on deviation of minimum boundary value has been used to eliminate the encoding error of $\mathrm{CGH}$. In the experiment, we use a liquid crystal spatial light modulator to generate CGHs and measure residuals of reconstructed wave-front of a secondary parabolic surface. The measurement results show that average decrease of the RMS values of the residuals is $0.07 \lambda$ when their periods range from 3 to 6 , which indicates the optimization method is effective.

[DOI: http://dx.doi.org/10.2971/jeos.2014.14039]

Keywords: Aspheric surface testing, computer-generated holograms, holographic encoding, liquid crystal spatial light modulator

\section{INTRODUCTION}

In the aspheric surface testing by computer-generated holography, computer-generated hologram (CGH) is usually used as a standard model to reconstruct the ideal wave-front, and further measuring the error existed on the aspheric surface. Compared with optical holography, computer-generated holography does not need a real standard aspheric surface as reference object during the measurement. Instead, computer-generated holography utilizes the mathematic model of the tested aspheric surface to generate its CGH correspondingly and prints the pattern onto a film or generates the pattern by optical devices such as liquid crystal spatial light modulator. This advantage makes computer-generated holography become a potential application with an attractive prospect [1]-[5]. The introduction of phase-only CGH in optical testing simplifies the optical path configuration and improves the diffraction efficiency [6]-[8]. However, during the encoding of CGH especially for CGH with multiphase levels, errors might occur through the process which cause phase mismatch in the generated hologram. Moreover, the error can be passed and amplified in measurement optical path and such accumulated error will further reduce the accuracy of reconstructed wave-front. However, the current research still focuses on how to correct or reduce alignment error of optical path, optical system error and wave-front reconstruction error exist in aspheric testing [9]-[12], but there is few research discussing encoding error.

In this article, we have studied how analyze encoding error generated during fabricating CGHs and correct this error. In the experimental part, a liquid crystal spatial light modulator has been applied to generate CGHs, and encoding errors before and after correction have been compared and analyzed to prove the effectiveness of our proposed optimization method.

\section{PRINCIPLE OF ENCODING FOR CGH}

According to the mathematic expression of the rotationally symmetric aspheric, its corresponding model $W(x, y)$ can be expressed as shown below,

$$
\begin{aligned}
W(x, y)= & \frac{c S^{2}}{1+\sqrt{1-(L+1) c^{2} S^{2}}} \\
& +A_{1} S^{4}+A_{2} S^{6}+\cdots+A_{n} S^{2 n}
\end{aligned}
$$

where $x, y$ is the Cartesian coordinate which corresponds to the bottom projection of any point on the aspheric surface and 
meets the condition $S^{2}=x^{2}+y^{2} . R_{0}$ is the radius of curvature of the surface and $c=\frac{1}{R_{0}} . A_{1}, A 2, \ldots, A_{n}$ are high order aspheric coefficients and $L$ is the eccentricity. To get a secondary aspheric surface let $A_{1}, A 2, \ldots, A_{n}=0, L=-e^{2}$.

We adopt phase type CGH-encoding method to process the holographic encoding for the aspheric surface $W(x, y)$. Firstly, $W(i, j)$ is obtained by sampling and discretizing $W(x, y)$ which can be expressed as,

$$
\begin{aligned}
& W(i, j) \\
= & {\left[W(x, y) \cdot \operatorname{comb}\left(\frac{x}{N}\right) \cdot \operatorname{comb}\left(\frac{y}{N}\right) \cdot \operatorname{rect}\left(\frac{x}{D}, \frac{y}{D}\right)\right] }
\end{aligned}
$$

where $i, j=1,2,3 \cdots N$ and $N$ is the sampling number, $D$ is the aperture diameter of the aspheric surface. Then, $W(i, j)$ is quantified modulo $2 \pi$ to generate a phase map. After amplification and rounding, the phase map can be transferred into a grayscale map whose value ranges from 0 to 255 . The grayscale map is called phase-only computer-generated hologram which is shown below,

$$
W_{C G H}(i, j)=\operatorname{round}\left[255 \cdot \frac{1}{\lambda h} \cdot \bmod [W(i, j) \cdot h]_{2 \pi}\right]
$$

Where $\lambda$ is light wavelength, $h$ is the wave vector and $h=\frac{2 \pi}{\lambda}$. The principle of phase type CGH-encoding is depicted in Figure 1.

Based on the knowledge of how a phase-only CGH is encoded, it is obvious to see that there exists encoding error during sampling, discretization and quantification processes due to rounding and value amplification, and the error is a kind of principal error.

\section{ANALYSIS AND CORRECTION OF ERROR}

\subsection{Analysis of encoding error of $\mathrm{CGH}$}

If $L$ in Eq. (1) is set to be -1 a mathematic model $W(x, y)$ of secondary parabolic surface can be obtained and its CGH $W_{C G H}(i, j)$ is also generated by expressions (2) and (3). Taking the CGHs quantified by 16 and 32 periods (Sampling rate $512 \times 512$ ) as our discussing object, we extract the central section to observe the grayscale distribution in that profile which is shown in Figure 2, where Figure 2(a) and Figure 2(b) give half grayscale distributions of CGHs quantified by 16 and 32 periods respectively. Theoretically, the initial value of each period should be 0 and the maximum value of each period should be 255, and the value of the gray scale should increase linearly from 0 to 255 . However, the real situation shows that nonlinear error exists in each period and distributes randomly. And the value of the nonlinear error increases when the period number gets larger. The analysis of the existed error indicates that there is encoding error in the phase-only CGH. Error correction can be used to avoid error amplification in aspheric surface testing and hence enhance the measuring accuracy.

When the sampling number is fixed, for example the sampling rate is set to be $512 \times 512$, the relationship between the RMS value of encoding error and quantization period is depicted

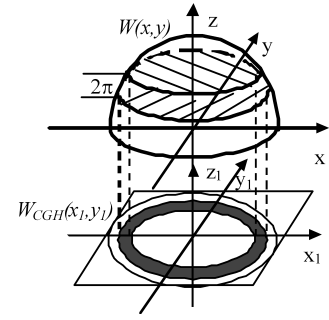

FIG. 1 Principle of phase type CGH-encoding for a secondary aspheric surface

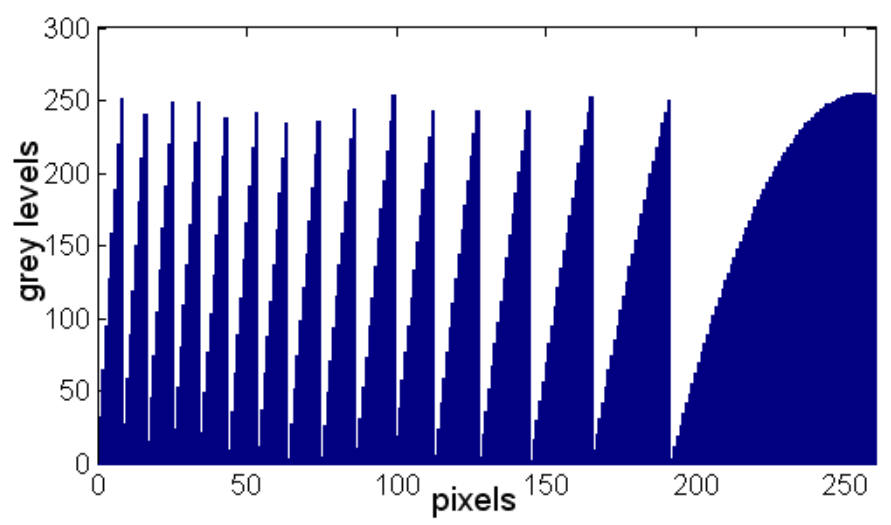

(a)

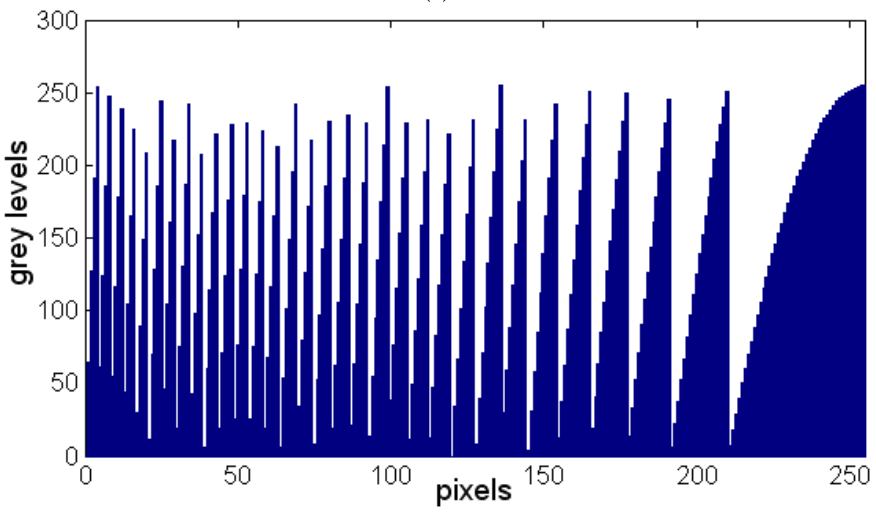

(b)

FIG. 2 Half grayscale distributions of CCHs with different quantization periods (Sampling rate $512 \times 512$ ) (a) Grayscale distributions of CGH with 16 periods; (b) Grayscale distributions of $\mathrm{CGH}$ with 32 periods.

in Figure 3. The RMS value of encoding error is defined as,

$$
e_{r m s}=\frac{1}{m} \sqrt{\sum_{i=1}^{m} \sum_{j=1}^{m}\left[W_{C G H}(i, j)-W_{0}(i, j)\right]^{2}}
$$

Where $W_{0}(i, j)$ is desired CGH and $\mathrm{m}$ is the maximum of sampling numbers. The encoding error of CGH steadily increases with the grow in quantization period. When the period number is 32 , the maximum rms of encoding error is 16.46 which will lead wave-front aberration $0.085 \lambda$.

\subsection{Correction of encoding error of $\mathrm{CGH}$}

An optimization method based on deviation of minimum boundary value is adopted to correct the existed error. Onedimension array $B_{1}, B_{2}, \cdots, B_{N}$ which goes through symmetry center point is extracted from $W_{C G H}(i, j)$. In the onedimension array, the minimum boundary value $b_{k}$ of each period is extracted. Through solving linear equations, the devi- 


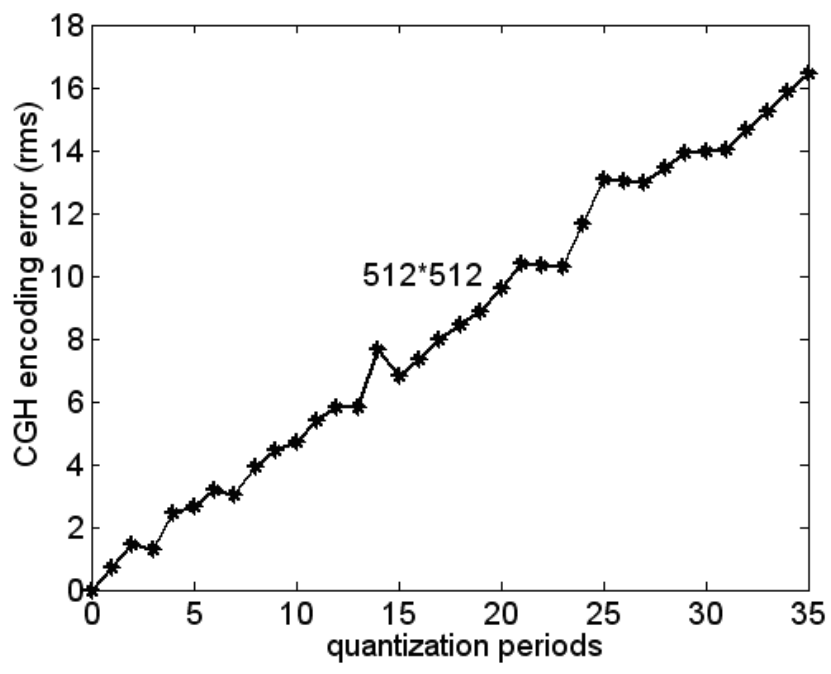

FIG. 3 Error distribution curve of CGH under $512 \times 512$ sampling rate.

ation of minimum boundary value of each period $z_{k}$ can be acquired.

$$
z_{k}=b_{k}-b_{0}
$$

where $b_{0}$ is the expectation value of the minimum grayscale value for one period and $k$ is the period number $k=1,2,3 \cdots C$ and $C$ is the maximum period number. The adjustment coefficient $\beta_{k}$ for zooming in or out grayscale values of each period in $W_{C G H}(i, j)$ can be obtained by solving the equation below,

$$
\beta_{k}=\frac{a_{0}}{a_{k}-z_{k}}-b_{0}
$$

Where $a_{k}$ is the maximum boundary value of each period and $a_{0}$ is the expectation value of the maximum grayscale value for one period. A CGH is composed of a square matrix with some data ring bands corresponding to quantization periods. The correction principle is to use $\beta_{k}$ to correct the data of the k-th ring band needed to be extracted by the boundary expression. After every ring band is corrected a new CGH is obtained by combining the corrected ring bands. The boundary expression is defined and created, and data $W_{k}(i, j)$ in each period is extracted from $W_{C G H}(i, j)$,

$$
W_{k}(i, j)= \begin{cases}W_{C G H}(i, j), & \lambda(k-1) \leq W(i, j) \leq \lambda k \\ 0, & W(i, j)<\lambda(k-1) \text { or } W(i, j)>\lambda k\end{cases}
$$

where $\lambda$ is the wavelength of the light. The corrected ring band $R_{k}(i, j)$ can be obtained by the expression below,

$$
R_{k}(i, j)=\left(1+\beta_{k}\right)\left[W_{k}(i, j)-z_{k}(i, j)\right]
$$

Where $z_{k}(i, j)$ is the matrix of the deviation of minimum boundary value which constituted by $z_{k}$ in the following rule,

$$
z_{k}(i, j)= \begin{cases}z_{k}, & \lambda(k-1) \leq W(i, j) \leq \lambda k \\ 0, & W(i, j)<\lambda(k-1) \text { or } W(i, j)>\lambda k\end{cases}
$$

The next step is to accumulate data of each ring band after adjustment, generating the corrected $W_{C G H 1}(i, j)$, which can be expressed as,

$$
W_{C G H 1}(i, j)=\text { round }\left(\sum_{k=1}^{C} R_{k}(i, j)\right)
$$

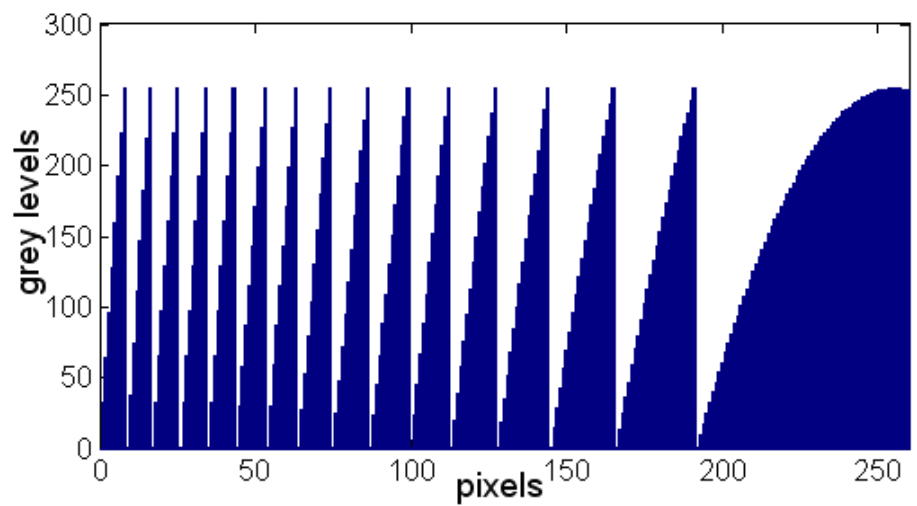

(c)

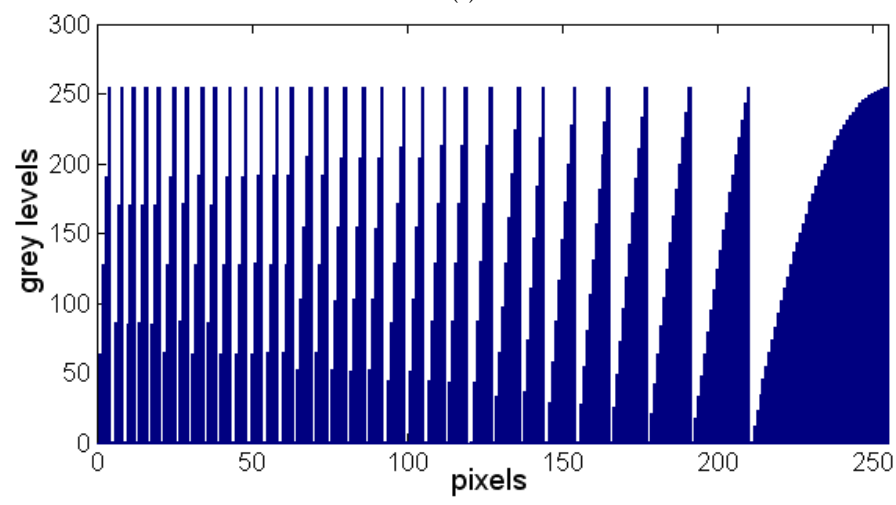

(d)

FIG. 4 Half grayscale distributions of CGHs with different quantization periods after correction (Sampling rate $512 \times 512$ ) (a) Grayscale distributions of CCH with 16 periods; (b) Grayscale distributions of CGH with 32 periods.

Finally, the corrected CGH should be checked by the condition whether it meets the setting requirement $\left|z_{k}\right|<z_{0}$. If the result is no, the corrected CGH will be substituted to Eq. (5) to start the loop from Eqs. (5) to (10). On the contrary, if the result meets the condition, corrected CGH is acquired.

\section{CORRECTION EXPERIMENT OF ENCODING ERROR}

The corrected CGHs quantified by 16 and 32 periods are shown in Figure 4. Compared with Figure 2, there is almost no error in CGHs because the minimum and maximum grayscale values can reach 0 and 255 respectively and the grayscale value in each periods distributes linearly. It is clear that the encoding error can be eliminated after the error correction above. Then the validation experiment is built by using the phase-only liquid crystal spatial light modulator (LCSLM) from BNS company to generate CGH and using AK100 Fizeau interferometer to measure the reconstructed wave-front. Using LCSLM to reconstruct wave-front of a secondary parabolic surface by loading the CGH patterns which range from 3 to 6 periods. The theoretical parabolic surface and the CGH quantified by 6 periods are shown in Figure 5. The errors of wavefronts reconstructed by the LCSLM before and after correction of encoding errors of CGH are listed and compared in Table 1. The RMS value of residual error on the parabolic surface can 


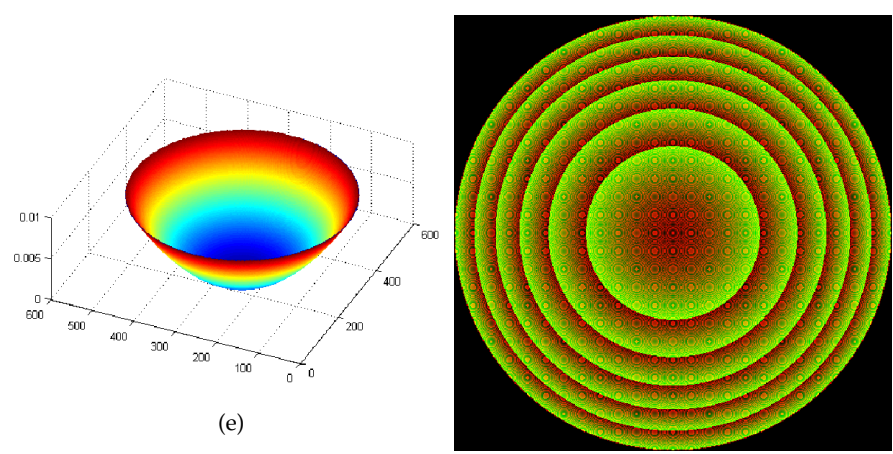

(f)

FIG. 5 Parabolic surface and its CGH (a) Parabolic surface; (b) $\mathrm{CGH}$ of parabolic surface.

\begin{tabular}{|c|c|c|c|c|}
\hline periods & 3 & 4 & 5 & 6 \\
\hline RMS uncorrected & 0.5991 & 0.7978 & 0.8296 & 0.7452 \\
\hline RMS corrected & 0.5125 & 0.7488 & 0.7232 & 0.6918 \\
\hline
\end{tabular}

TABLE 1 Residual error of wave-front.

be calculated by expression below,

$$
\delta_{r m s}=\frac{1}{m} \sqrt{\sum_{i=1}^{m} \sum_{j=1}^{m}\left[P_{\text {mea }}(i, j)-P_{0}(i, j)\right]^{2}}
$$

Where $P_{0}(i, j)$ is ideal wave-front phase, $P_{\text {mea }}(i, j)$ is measured value and $m$ is the maximum of sampling numbers. The RMS has experienced an $0.07 \lambda$ ( $\lambda$ is $632.8 \mathrm{~nm}$ ) average decrease after error correction.

There are a few factors that cause the residual error to be larger than expected. Firstly, the distortion existed in the optical measuring system has not been corrected. Secondly, the resolution of the phase pattern from the measurement is only $130 \times 130$ pixels which is far smaller than the resolution of $512 \times 512$ pixels of CGH from LCSLM. In that case, interpolation should be used which might bring error in some extent. Figure 6 gives the measurement result of CGH quantified by 5 periods. Figure 6(a), 6(b), 6(c) and Figure 6(d), 6(e), 6(f) show the results before and after error correction respectively (the sampling rate is set to be $512 \times 512$ pixels). Because the aperture of reference mirror in the interferometer is $150 \mathrm{~mm}$ and the working aperture of the LCSLM is $7.68 \mathrm{~mm}$ which is far different from the former's dimension, plane mirror should be more facilitated to be used to adjust the optical path. However, when the quantified period exceeds 6 periods, the parabolic surface and the reference plane mirror cannot form interference pattern properly which limits the setting for the period number to be under 6 periods. Based on the principle of encoding error correction mentioned above, when the period number is small, the error is small too. Therefore, the improvement after error correction is not significant when the period is set to be 5 in Figure 6. Facing this limitation, our next plan is to redesign the optical part to meet the need of setting larger quantified period.

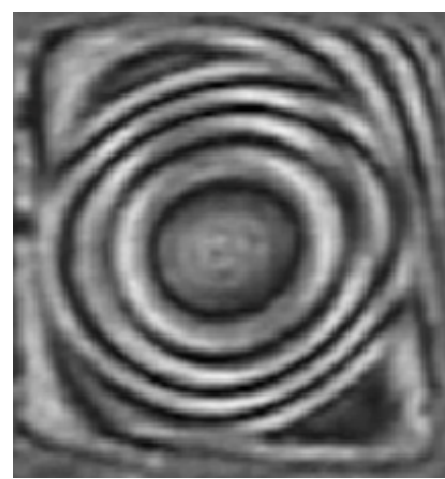

(g)

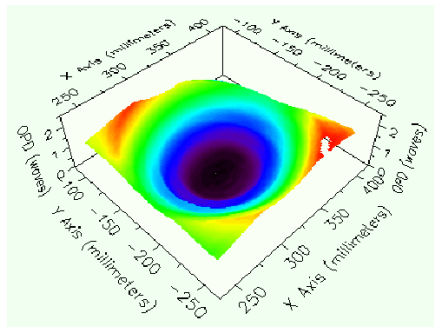

(h)

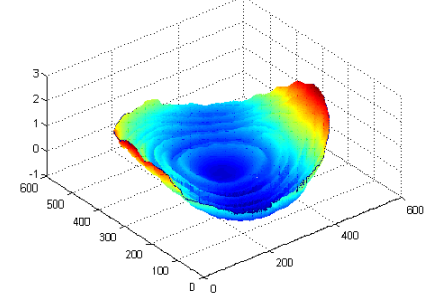

(i)

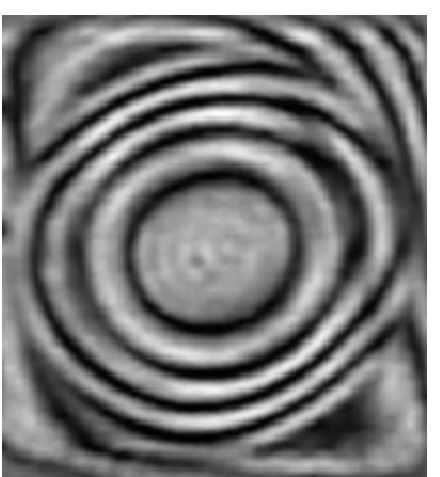

(j)

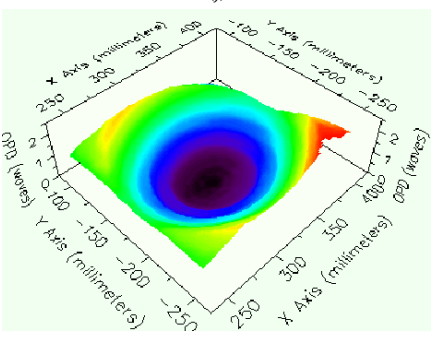

(k)

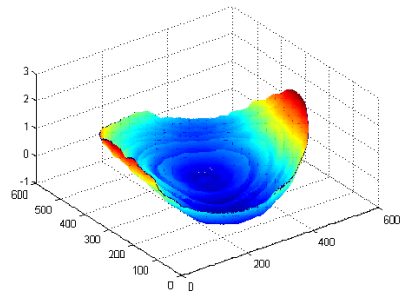

(1)
FIG. 6 Measurement results of encoding error of CGH quantified by 5 periods (a) Interference fringe of before correction; (b) $3 \mathrm{D}$ wave-front; (c) Residual error (d) Interference fringe of after correction; (e) $3 \mathrm{D}$ wave-front; ( $f$ ) Residual error

\section{CONCLUSIONS}

In this article, we use phase type CGH-encoding to generate CGHs for secondary parabolic surface. The analysis of encoding error indicates that the error will increase with the growth of the quantified period number. For CGH with $512 \times 512$ sampling rate, when the period reaches 32 the maximum of encoding error is 16.46 which can lead wave-front aberration $0.085 \lambda$. In order to block the transmission of error in aspheric surface testing, an optimization method based on deviation of minimum boundary value has been proposed. In the experiment, LCSLM is loaded CGH with 3,4,5,6 periods to reconstruct secondary parabolic surface. After correction, RMS value of the residual error of the parabolic surface meets a $0.07 \lambda$ average decrease. The experimental results validates the effectiveness of our proposed method for encoding error correction. The combination of CGH encoding and our encoding error correction method can realize high accuracy aspheric surface testing.

\section{ACKNOWLEDGMENTS}

This research is supported by National Natural Science Foundation of China (NO. 61178069) and Natural Science Foundation of Heilongjiang Province of China (NO. F201117). 


\section{References}

[1] H. Liu, Z. W. Lu, F. Y. Li, and Q. Sun, "Design of a novel hologram for full measurement of large and deep convex aspheric surfaces," Opt. Express 15, 3120-3126 (2007).

[2] E. Garbusi, and W. G. Osten, "Analytical study of disturbing diffraction orders in in-line computer generated holograms for aspheric testing," Opt. Commun. 283, 2651-2656 (2010).

[3] X. M. Hong, P. J. Gou, and J. F. Ren, "Two Computer-generated Holograms for Testing Convex Aspheric Surface," Proc. SPIE 8192, 1-8 (2011).

[4] M. Kino, and M. Kurita, "Interferometric testing for off-axis aspherical mirrors with computer-generated holograms," Appl. Optics 51, 4291-4297 (2012).

[5] A.G. Poleshchuk, R. K. Nasyrov, and J. M. Asfour, "Combined computer-generated hologram for testing steep aspheric surfaces," Opt. Express 17, 5420-5425 (2009).

[6] S. Reichelt, C. Pruss, and H. J. Tiziani, "Absolute interferometric test of aspheres by use of twin computer-generated holograms," Appl. Optics 42, 4468-4479 (2003).
[7] V. Arrizón, U. Ruiz, R. Carrada, and L. A. González, “Pixelated phase computer holograms for the accurate encoding of scalar complex fields," J. Opt. Soc. Am. A 24, 3500-3507 (2007).

[8] S. Peterhänsel, C. Pruss, and W. Osten, "Phase errors in high line density CGH used for aspheric testing: beyond scalar approximation," Opt. Express 21, 11638-11651 (2013).

[9] Y. Tang, X. Y. Su, Y. K. Liu, and H. L. Jing, “3D shape measurement of the aspheric mirror by advanced phase measuring deflectometry," Opt. Express 16, 1590-1596 (2008).

[10] W. R. Cai, P. Zhou, C. Y. Zhao, and J. H. Burge, "Analysis of wavefront errors introduced by encoding computer-generated holograms," Appl. Optics 52, 8324-8331 (2013).

[11] F. Z. Li, J. L. Zhao, R. G. Li, B. Z. Zhang, L. G. Zheng, and X. J.Zhang, "Design and Fabrication of $\mathrm{CGH}$ for aspheric surface testing and its experimental comparison with null lens," Proc. SPIE 7656, 1-6 (2010).

[12] H. S. Yang, J. B. Song, I.W. Lee, and Y. W. Lee, "Testing of steep convex aspheric surface with a Hartmann sensor by using a $\mathrm{CCH}^{\prime}$, Opt. Express 14, 3247-3254 (2006). 